\title{
Loss of GATA5 expression due to gene promoter methylation induces growth and colony formation of hepatocellular carcinoma cells
}

\author{
LEI XIA ${ }^{1 *}$, YAN GONG $^{2 *}$, AIQUN ZHANG $^{3}$, SHOUWANG CAI $^{3}$ and QIANG ZENG ${ }^{2}$ \\ ${ }^{1}$ Department of Medical Security; ${ }^{2}$ Health Management Institute; ${ }^{3}$ Department of Hepatobiliary Surgery, \\ Chinese People's Liberation Army General Hospital, Beijing 100853, P.R. China
}

Received October 24, 2014; Accepted August 18, 2015

DOI: $10.3892 / 01.2015 .3974$

\begin{abstract}
GATA5 is a transcription factor that is capable of suppressing the development of various types of human cancer. The present study investigated the expression of GATA5 and GATA4, and examined their roles in the proliferation and colony formation ability of hepatocellular carcinoma (HCC) tissues and cells. The GATA4 and GATA5 expression levels and gene promoter methylation of HCC tissue samples from 38 patients and HCC cell lines were analyzed using reverse transcription-polymerase chain reaction (RT-PCR) and methylation-specific PCR (MSP), respectively. The effects of GATA4 and GATA5 overexpression on the proliferation and colony forming ability of HCC cells were also assessed using cell viability and colony formation assays. A luciferase reporter assay was utilized to investigate the transcriptional interaction of GATA4 and GATA5 with canonical Wnt signaling. The results indicated that the expression levels of GATA4 and GATA5 were lost or reduced following methylation of gene promoters in HCC tissues and cell lines. Treatment with a demethylating agent, 5-aza-2'-deoxycytidine (5-AZA), restored GATA4 and GATA5 expression in HCC cell lines. Furthermore, methylation of the GATA5 promoter was observed to be associated with the age of patients exhibiting HCC. Restoration of GATA4 and GATA5 expression inhibited colony formation and induced apoptosis of HCC cells in vitro. The present study concluded that the expression levels of GATA4 and GATA5 were reduced in HCC tissues and cell lines. Treatment with 5-AZA restored GATA4 and GATA5 expression in HCC cell lines, suppressing tumor cell growth and colony formation, as well as inducing apoptosis.
\end{abstract}

Correspondence to: Professor Qiang Zeng, Health Management Institute, Chinese People's Liberation Army General Hospital, 28 Fuxing Road, Beijing 100853, P.R. China

E-mail: zq301@hotmail.com

*Contributed equally

Key words: GATA5, expression, methylation, proliferation, cloning formation, hepatocellular carcinoma

\section{Introduction}

Primary liver cancer is the fifth most common cause of cancer-associated mortality worldwide (1), and up to $90 \%$ of primary liver cancers are hepatocellular carcinomas (HCC) (2). Risk factors for hepatocarcinogenesis include chronic hepatitis $\mathrm{B}$ or $\mathrm{C}$ infection, alcohol consumption and aflatoxin B1 contamination (2). HCC development, similarly to that of other types of cancer, is a multistep process, involving numerous genetic and epigenetic alterations (3). It has been reported that promoter methylation of certain tumor suppressor genes occurs frequently during HCC development and progression $(4,5)$.

The GATA gene family comprises a group of zinc finger transcription factors that are able to bind to the GATA motif (WGATAR), which is present in the promoters of certain genes (6). GATA1, GATA2 and GATA3 are functionally involved in cellular lineage determination (7), while GATA4, GATA5 and GATA6 regulate the development of endoderm-derived organs, including the heart and gut (8). During development, GATA4 and GATA5 induce differentiation of embryonic stem cells into specific, mature gut cells (9). In adults, GATA4 and GATA5 regulate epithelial cell differentiation $(10,11)$. However, altered expression of GATA4 and GATA5 proteins is associated with tumorigenesis in certain organs and tissues, including gastric and colon cancer; thus, GATA4 and GATA5 may act as putative tumor suppressor genes $(11,12)$. A previous study revealed that GATA4 and GATA5 expression was lost in ovarian and gastric cancer, and demonstrated that chromosomal regions of GATA4 (8p23.1-p22) and GATA5 (20q13.2-q13.3) loci were frequently deleted in various types of human cancer (13). Additional studies identified that loss of GATA4 and GATA5 expression during human neoplastic progression, including that of pancreatic, non-small cell lung, esophageal and renal cancer, was due to promoter methylation (14-16). Loss of GATA4 and GATA5 expression may also alter the typical expression patterns of numerous downstream gene networks with antineoplastic properties (11). For example, GATA transcription factors are able to integrate Wnt signaling during heart muscle formation $(17,18)$ and alteration of the $\mathrm{Wnt} / \beta$-catenin pathway has a critical role in the development of various types of human cancer, including HCC (19). 
Thus, in the present study, the expression levels and promoter hypermethylation of GATA4 and GATA5 were evaluated in human HCC tissue specimens and cell lines. The effects of GATA4 and GATA5 expression restoration in HCC cells in vitro were subsequently characterized.

\section{Materials and methods}

Cell lines and culture. Human liver cancer cell lines (HepG2, SMZ7721, HBxF344, PLC/PRF/5, SK-Hepl, 97H and 7402) and an SV-40-immortalized Lo-2 liver cell line were obtained from the Cell Bank of the Chinese Academy of Sciences (Shanghai, China). Cells were maintained in RPMI-1640 medium (Grand Island Biological Company, Grand Island, NY ,USA) or Dulbecco's modified Eagle medium (DMEM; Invitrogen Life Technologies, Carlsbad, CA, USA), supplemented with $10 \%$ fetal bovine serum (FBS; Grand Island Biological Company) in a humidified incubator, with $5 \% \mathrm{CO}_{2}$ and $95 \%$ air, at $37^{\circ} \mathrm{C}$. Cells were passaged with trypsin (Sigma-Aldrich, St. Louis, MO, USA) into T-75 flasks (Sarstedt, Nümbrecht, Germany) using a 1:3 split when $80 \%$ confluence was achieved $\left(\sim 1 \times 10^{6}\right.$ cells/flask).

For treatment with 5-aza-2'-deoxycytidine (5-AZA; Sigma-Aldrich), cells were separated and cultured at a low density ( $30 \%$ confluence) overnight and subsequently treated with 5-AZA ( $1 \mu \mathrm{mol} / \mathrm{l})$ for $96 \mathrm{~h}$. Growth medium was replaced every $24 \mathrm{~h}$, and at the conclusion of treatment, DNA and RNA was isolated as described below.

HCC tissue specimens. A total of 38 surgically resected and pathologically confirmed HCC specimens ( 8 females and 30 males; aged 30-79 years) were obtained from the Chinese People's Liberation Army General Hospital (Beijing, China) between January 2009 and June 2011 and stored in liquid nitrogen prior to use. The present study was approved by the Institutional Review Board of the Chinese People's Liberation Army General Hospital. Written informed consent was obtained from each patient or their guardian. Tumor and paired adjacent tissue samples $(\geq 1 \mathrm{~cm}$ away from tumor lesion) were collected. Tumor staging was determined according to the American Joint Committee on Cancer Cancer Staging Manual, 2010 (7th Edition) (20). Normal hepatic tissue was obtained from the edge of resected hemangiomas of the liver.

DNA extraction. HCC cell lines were cultured and treated with 5-AZA as described and subsequently digested with 0.5\% trypsin-EDTA (Sangon Biotech Co., Ltd). Following digestion, DNA was extracted using the proteinase-K method. Genomic DNA from normal and HCC liver tissues was also extracted using the proteinase-K method (21). DNA was dissolved in Tris-EDTA buffer and stored at $-20^{\circ} \mathrm{C}$ until use.

$R N A$ isolation and semi-quantitative reverse transcription-polymerase chain reaction (RT-PCR). Total cellular RNA was isolated from the cells using TRIzol reagent (Invitrogen Life Technologies) according to the manufacturer's instructions. RNA quality and quantity was assessed using agarose gel electrophoresis (1\%) and spectrophotometric analysis using a $260-280$ ratio. RNA was stored at $-80^{\circ} \mathrm{C}$ until use. First strand complementary DNA (cDNA) was synthesized with oligo-(dT) primers using a PrimeScript ${ }^{\mathrm{TM}}$ 1st Strand cDNA Synthesis kit obtained from Invitrogen Life Technologies. RNA $(2 \mu \mathrm{g})$ was subjected to first strand cDNA synthesis, and subsequently $1 \mu \mathrm{l} \mathrm{cDNA}$ from the RT reaction was subjected to PCR amplification in a total reaction volume of $25 \mu \mathrm{l}$. PCR amplification was performed using primer sets derived from the published GATA4 and GATA5 gene sequences (22). PCR conditions were set to $94^{\circ} \mathrm{C}$ for $1 \mathrm{~min}, 64^{\circ} \mathrm{C}$ for $1 \mathrm{~min}$ and $72^{\circ} \mathrm{C}$ for $90 \mathrm{sec}$ for 3 cycles and $94^{\circ} \mathrm{C}$ for $1 \mathrm{~min}, 61^{\circ} \mathrm{C}$ for $1 \mathrm{~min}$ and $72^{\circ} \mathrm{C}$ for $90 \mathrm{sec}$ for additional 3 cycles, and $94^{\circ} \mathrm{C}$ for $1 \mathrm{~min}, 58^{\circ} \mathrm{C}$ for $1 \mathrm{~min}$, and $72^{\circ} \mathrm{C}$ for $90 \mathrm{sec}$ for final 3 cycles and then $94^{\circ} \mathrm{C}$ for $1 \mathrm{~min}, 55^{\circ} \mathrm{C}$ for $1 \mathrm{~min}$, and $72^{\circ} \mathrm{C}$ for $90 \mathrm{sec}$ for 23 cycles and final $72^{\circ} \mathrm{C}$ extension for 7 min. GATA4 primers used were as follows: Sense, 5'-CTGGCCTGTCATCTCACTACG-3' and antisense, 5'-GGTCCGTGCAGGAATTTGAGG-3'. GATA5 primers used were as follows: Sense, 5'-TCGCCAGCA CTGACAGCTCAG-3' and antisense, 5'-TGGTCTGTTCCA GGCTGTTCC-3'. A total of 32 PCR cycles (based on preliminary investigation) were performed for semi-quantitative measurement of GATA4 and GATA5 gene expression. GAPDH was amplified for 25 cycles as an internal control, to ensure equal loading, as well as cDNA quality and quantity. GAPDH primers were as follows: Sense, 5'-GACCACAGTCCATGCCATCAC-3' and antisense, 5'-GTCCACCACCCTGTTGCTGTA-3'. PCR products were electrophoresed in $1.5 \%$ agarose gels containing ethidium bromide and were evaluated using UV light.

Methylation-specific PCR (MSP). Genomic DNA from HCC tissues and cell lines was modified using bisulfite modification as described previously (23). MSP primers were designed according to the genomic sequences surrounding the putative transcription start sites of the GATA4 and GATA5 genes. Primer sequences were synthesized by Sangon Biotech Co.,Ltd. (Shanghai, China) to allow MSP to detect bisulfite-induced changes affecting unmethylated and methylated alleles. MSP primers were synthesised by Shanghai Sangon Biotech Co., Ltd; MSP primers for GATA4 were as follows: Methylated sense, 5'-GTATAGTTTCGTAGTTTGCGTTTA GC-3' and antisense, 5'-AACTCGCGACTCGAATCCCCG-3'; unmethylated sense, 5'-TTTGTATAGTTTTGTAGTTTGTGT TTAGT-3' and antisense, 5'-CCCAACTCACAACTCAAATCC CCA-3'. MSP primers for GATA5 were as follows: Methylated sense, 5'-AGTTCGTTTTTAGGTTAGTTTTCGGC-3' and antisense, 5'-CCAATACAACTAAACGAACGAACCG-3'; unmethylated sense, 5'-TGGAGT TTGTTTTTAGGT TAGTTTTTGGT-3' and antisense,5'-CAAACCAATACAACT AAACAAACAAACCA-3'. Each MSP reaction incorporated $\sim 100$ ng bisulfite-treated DNA, $25 \mathrm{pM}$ each primer, $100 \mathrm{pM}$ deoxynucleotides, $2.5 \mu \mathrm{l}$ 10X PCR buffer and 1 unit JumpStart Red Taq Polymerase (Sigma-Aldrich) in a final reaction volume of $25 \mu$ l. PCR cycling conditions were as follows: $95^{\circ} \mathrm{C}$ for $5 \mathrm{~min}$, followed by 35 cycles at $95^{\circ} \mathrm{C}$ for $30 \mathrm{sec}, 60^{\circ} \mathrm{C}$ for $30 \mathrm{sec}$ and $72^{\circ} \mathrm{C}$ for $30 \mathrm{sec}$. MSP products were analyzed using $2 \%$ agarose gel electrophoresis. Subsequently, MSP products were subcloned into a pGEM $^{\circledR}-\mathrm{T}$ vector (Promega Corp., Madison, WI, USA), and transformed into Escherichia coli DH5 $\alpha$ cells (Invitrogen Life Technologies). Candidate plasmid clones were sequenced by Sangon Biotech Co., Ltd. 
Expression vector and gene transfection. Full-length GATA4 and GATA5 cDNA were purchased from Orignene Technologies, Inc. (Rockville, MD, USA) and subcloned into pCMV6-XL4 (OriGene Technologies, Inc.) in Escherichia coli DH5 $\alpha$ cells (Invitrogen Life Technologies). Candidate plasmid clones were sequenced by Sangon Biotech Co., Ltd. Following amplification and sequence confirmation, these vectors were named pCMV-GATA4 and pCMV-GATA5. For gene transfection, HepG2 cells were seeded in DMEM containing 10\% FBS overnight. On the following day, cells were transfected with pCMV-GATA4 or pCMV-GATA5 using Lipofectamine ${ }^{\circledR} 2000$ (Invitrogen Life Technologies) according to the manufacturer's instructions. Twenty-four hours later, the cells were separated into fresh dishes, at a 1:3 ratio, and treated with $400 \mu \mathrm{g} / \mathrm{ml}$ gentamycin sulfate (G418; Invitrogen Life Technologies). Cell culture media was replaced every three days. Following three weeks of incubation, the cells formed stable gene-transfected clones and were used for the following experiments.

Protein extraction and immunoblot assay. Total protein was prepared from the vector control (pCMV-con vector only) and pCMV-GATA4 (pc-GATA4) or pCMV-GATA5 (pc-GATA5)-transfected HepG2 cells, with ice-cold lysis buffer (Beyotime Institute of Biotechnology, Haimen, China), according to the manufacturer's instructions. Following centrifugation at $12,000 \mathrm{x} \mathrm{g}$ for $15 \mathrm{~min}$ at $4^{\circ} \mathrm{C}$, supernatants were collected and protein concentration was assessed using a bicinchoninic acid protein assay kit (Beyotime Institute of Biotechnology). Equal quantities of protein were separated using 10\% SDS-PAGE and transferred onto polyvinylidene difluoride membranes (EMD Millipore, Billerica, MA, USA). Western blot analysis was performed using mouse monoclonal IgG2a anti-GATA4 (sc-25310, 1:400, Santa Cruz Biotechnology, Inc., Dallas, TX, USA) and rabbit monoclonal anti-GATA5 (G8669, 1:600, Sigma-Aldrich), while anti- $\beta$-actin antibody served as the loading control (Wuhan Boster Biological Technology, Ltd., Wuhan, China): the membranes were incubated overnight at $4^{\circ} \mathrm{C}$. Then the membranes were incubated with a horseradish peroxidase conjugated-goat anti-rabbit or mouse IgG (Shanghai Westang Bio Tech Co., Ltd, China). Chemiluminescence substrate solution (Pierce Biotechnology, Inc., Rockford, IL, USA) was applied to the membranes and X-ray film (Eastman Kodak Co., Rochester, NY, USA) was used to visualize protein bands.

Cell viability MTS assay. Cells transfected with a vector control, and pc-GATA4 or pc-GATA5, were seeded at a density of 500 cells/well in 96-well plates, and grown for 6 days prior to commencement of the cell proliferation assay. Cell proliferation was determined using cell counting kit-8 solution (Beyotime Institute of Biotechnology), according to the manufacturer's instructions. The optical density was measured at $492 \mathrm{~nm}$ using an Multiskan TM FC ELISA plate reader (ThermoFisher Scientific, Inc., Waltham, MA, USA). Experiments were performed in triplicate and repeated three times.

Flow cytometric apoptosis assay. HCC cells were transfected with a vector-control and pc-GATA4 or pc-GATA5 for $72 \mathrm{~h}$; subsequently, attached and floating cells were harvested and fixed using $70 \%$ ethanol for at least $48 \mathrm{~h}$. Cells were resuspended in $50 \mu \mathrm{g} / \mathrm{ml}$ propidium iodide (Sigma-Aldrich)and $100 \mu \mathrm{g} / \mathrm{ml}$ RNase (Takara Biotechnology Inc.) for $30 \mathrm{~min}$, and analyzed using flow cytometry (FACSCalibur ${ }^{\mathrm{TM}}$; BD Biosciences, Franklin Lakes, NJ, USA).

Colony formation assay. HepG2 cells were plated in 6-well culture plates, grown for $24 \mathrm{~h}$, and subsequently transfected with a vector control and pc-GATA4 or pc-GATA5 using FuGENE $^{\circledR} 6$ transfection reagent (Roche Diagnostics, Indianapolis, IN, USA) according to the manufacturer's instructions. Following $24 \mathrm{~h}$ of incubation, transfected cells were diluted and reseeded into fresh dishes at 500 cells/well in 6-well culture plates, and were subsequently grown and selected in $400 \mathrm{mg} / \mathrm{ml} \mathrm{G} 418$. Following a further 14 days of incubation, cells were fixed using $75 \%$ ethanol for $30 \mathrm{~min}$ and stained with $0.2 \%$ crystal violet (Beijing Zhongshan Biotechnology Co., Ltd, Beijing, China) for visualization and counting. The experiment was performed in triplicate and repeated once.

Luciferase reporter assay. HepG2 cells were plated at a density of $5 \times 10^{4}$ cells/well in 24-well culture plates and grown for $24 \mathrm{~h}$. Subsequently, the TOP/FOP flash system reporter construct (Promega Corporation, Madison, WI, USA) pGL3-OT $(0.4 \mu \mathrm{g})$ and pc-GATA4 or pc-GATA5 $(0.4 \mu \mathrm{g})$ were transfected into the HepG2 cells using FuGENE ${ }^{\circledR} 6$ transfection reagent according to the manufacturer's instructions. The pGL3-OT plasmid is a TCF/LEF-responsive reporter that contains three consensus TCF binding sites fused to the firefly luciferase gene. The pRL-TK vector served as the system internal control. Following $48 \mathrm{~h}$ of incubation, relative luciferase activity was measured using a GloMax ${ }^{\circledR}$ luminometer (Promega Corporation), and normalized for background Renilla luciferase activity using the Dual Luciferase Reporter Assay system (Promega Corporation), according to the manufacturer's instructions. For each experiment, the luciferase assay was performed three times.

Statistical analysis. Data are expressed as the mean \pm standard error where appropriate. All statistical analyses were performed using SPSS 13.0 for Windows (SPSS, Inc., Chicago, IL, USA). P-values for dichotomous variables were two-tailed and based on the Pearson $\chi^{2}$ test or the Pearson $\chi^{2}$ test with continuity correction. Continuous variables were analyzed using the Student's t-test. $\mathrm{P}<0.05$ was considered to indicate a statistically significant difference.

\section{Results}

GATA4 and GATA5 expression is lost due to gene promoter methylation in certain HCC cell lines. The HepG2, SMZ7721 and 7402 cell lines expressed undetectable levels of GATA4 mRNA, while PLC/PRF/5 cells expressed reduced levels of GATA4 mRNA. By contrast, $97 \mathrm{H}$ and Lo-2 cell lines expressed increased levels of GATA4 mRNA. Similarly, GATA5 mRNA was not detectable in PLC/PRF/5, HBfX344, Sk-hepl and SMZ7721 cell lines, whereas the HepG2, 97H, 7402 and Lo-2 cell lines expressed high levels of GATA5 mRNA (Fig. 1A). In order to investigate the underlying mechanisms responsible for the reduced expression of GATA4 

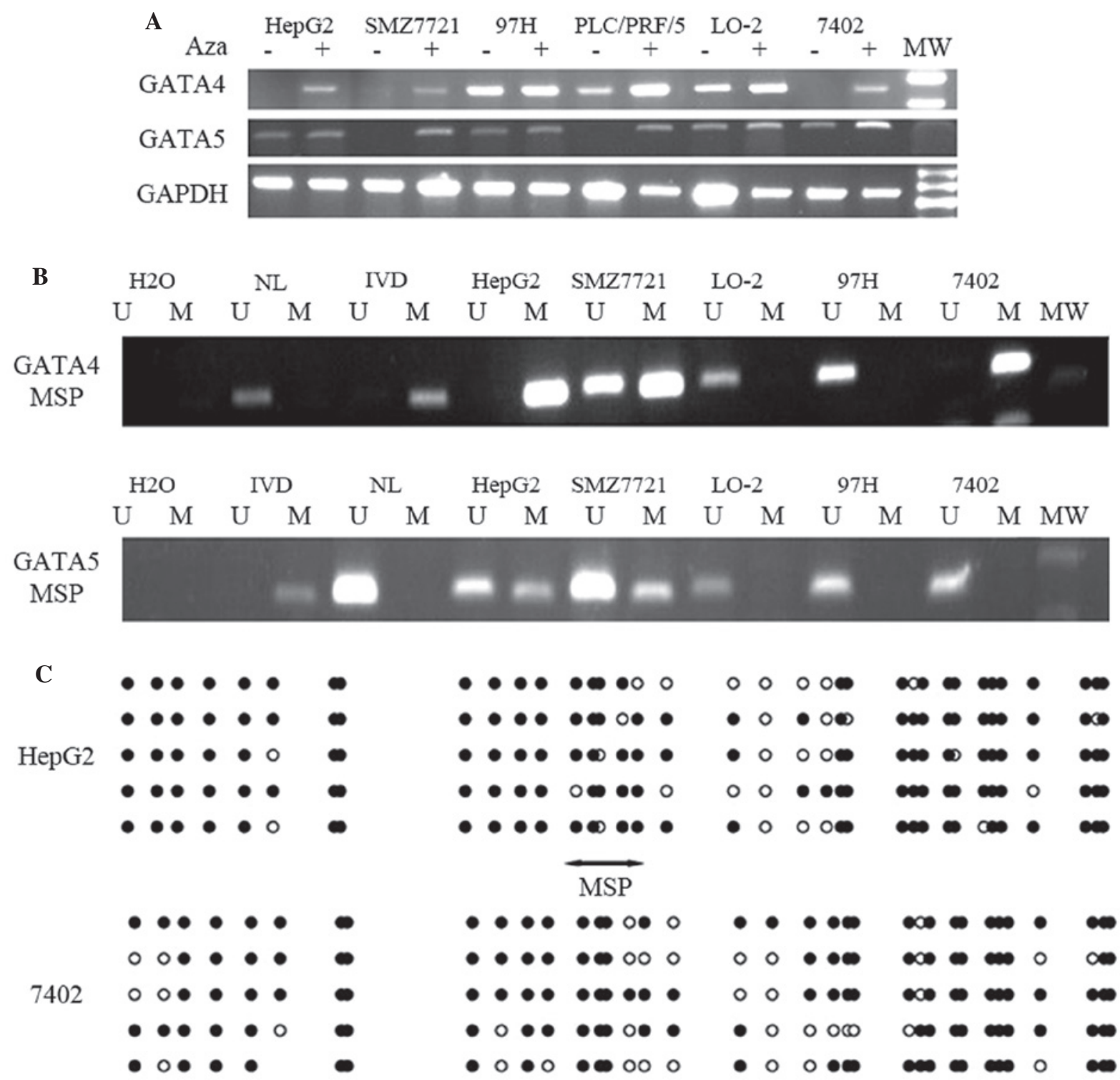

Figure 1. GATA4 and GATA5 expression is downregulated in HCC cell lines due to DNA methylation. (A) HCC cell lines and an immortal liver cell line (Lo-2) were cultured and treated with or without $10 \mu \mathrm{mol} / 15$-aza-2'-deoxycytidine for up to $96 \mathrm{~h}$. Reverse transcription-PCR analysis of GATA4 and GATA5 mRNA expression was performed. GAPDH mRNA served as an internal control. (B) MSP analysis of GATA4 and GATA5 promoter methylation in HCC cell lines. In vitro methylated DNA and DNA from normal human peripheral lymphocytes were used as methylated and unmethylated controls, respectively. (C) Sequence analysis of the GATA4 gene promoters following bisulfite modification in HCC cells. Black circles indicate methylated cytosine and white circles indicate unmethylated cytosine in the dinucleotide CpG. The region of the CpG island studied by MSP (doubleheaded arrow) spanned $142 \mathrm{bp}$. Bisulfite sequencing focused on a 385 bp ( +47 to +432 bp) CpG island downstream of the GATA4 transcription start site. H2O, water control; U, unmethylated alleles; $\mathrm{M}$, methylated alleles; HCC, hepatocellular carcinoma; IVD, in vitro methylated DNA; NL, normal human peripheral lymphocytes; PCR, polymerase chain reaction; MW, molecular weight; MSP, methylation-specific polymerase chain reaction.

and GATA5 in the aforementioned HCC cell lines, these cells were treated with a DNA-demethylating agent (5-AZA) and the expression of GATA4 and GATA5 was subsequently assessed. The results of the present study revealed that GATA4 expression was induced in HepG2 and 7402 cells, and GATA5 expression was significantly enhanced in SMZ7721 and PLC/PRF/5 cells following treatment with 5-AZA (Fig. 1A; $\mathrm{P}<0.05)$.

Furthermore, MSP analysis was performed to determine gene promoter methylation. The GATA4 promoter was entirely methylated in 7402 cells, as evidenced by undetectable GATA4 mRNA expression levels. Partial GATA4 promoter methylation was observed in HepG2 and SMZ7721 cells; HepG2 and SMZ7721 cells possessed methylated and unmethylated alleles (Fig. 1B). GATA4 expression was inversely associated with the levels of gene promoter methylation in the remaining HCC cell lines (Fig. 1A and B; Table I). Similarly, the GATA5 promoter was entirely methylated in PLC/PRF/5 cells, which exhibited undetectable levels of GATA5 mRNA. The GATA5 promoter was partially methylated in HepG2 and SMZ7721 cells, which possessed methylated and unmethylated alleles (Fig. 1B; Table I). In the present study, MSP products were cloned and sequenced and it was revealed that GATA4 promoter methylation in HepG2 and 7402 cell lines was present in the $\mathrm{CpG}$ islands of the gene promoter region (Fig. 1C).

Methylation of the GATA5 promoter is associated with increased age of patients with $\mathrm{HCC}$. To confirm that GATA4 
Table I. Expression and gene promoter region methylation of GATA4 and GATA5 in HCC cell lines.

\begin{tabular}{lccc}
\hline A, GATA4 & & & \\
\hline HCC cell line & RT-PCR & +5 -AZA & MSP \\
\hline HepG2 & - & -+ & M \\
SMZ7721 & - & U/M \\
HBfX344 & + & + & $\mathrm{U}$ \\
PRC/PLC/5 & + & + & $\mathrm{U}$ \\
97H & + & + & $\mathrm{U}$ \\
7402 & - & + & $\mathrm{M}$ \\
Sk-hepl & + & + & $\mathrm{U}$ \\
Lo-2 & + & + & $\mathrm{U}$ \\
\hline
\end{tabular}

\section{B, GATA5}

\begin{tabular}{lccc}
\hline HCC cell line & RT-PCR & $+5-A Z A$ & MSP \\
\hline HepG2 & -+ & -+ & U/M \\
SMZ7721 & - & -+ & U/M \\
HBfX344 & - & - & $\mathrm{U}$ \\
PRC/PLC/5 & - & + & $\mathrm{M}$ \\
97H & + & + & $\mathrm{U}$ \\
7402 & + & + & $\mathrm{U}$ \\
Sk-hepl & - & + & $\mathrm{M}$ \\
Lo-2 & + & + & $\mathrm{U}$ \\
\hline
\end{tabular}

RT-PCR, reverse transcription-polymerase chain reaction; MSP, methylation-specific PCR; 5-AZA, 5-Aza-2'-deoxycytidine; -, no expression; - +, partial expression; +, expression; U, unmethylated; M, methylated; U/M, partially methylated; HCC, hepatocellular carcinoma.

and GATA5 expression was lost due to gene promoter methylation in HCC cells ex vivo, HCC tissue samples were collected and GATA4 and GATA5 promoter methylation was analyzed. Specifically, MSP was performed on 38 samples of human HCC and surrounding non-tumor tissue obtained from patients with HCC. MSP GATA4 experiments were only successful for $28 \mathrm{HCC}$ and 26 non-tumor tissue specimens, whereas MSP experiments for GATA5 were succesful for all $38 \mathrm{HCC}$ and surrounding non-tumor tissue samples; the results revealed GATA4 and GATA5 promoter methylation in 2/28 (7.14\%) and 13/38 (34.20\%) HCC tissues and 3/26 (11.54\%) and 0/38 (0\%) surrounding non-tumor tissues, respectively (Fig. 2A and B). For GATA4 methylation, there was no statistically significant difference observed between $\mathrm{HCC}$ tissues and the surrounding tissue $(\mathrm{P}=0.578)$. For GATA5, methylation was only observed in tumor tissue.

Methylation of the GATA5 promoter was subsequently observed to be correlated with certain clinicopathological features. Notably, methylation of the GATA5 promoter in $\mathrm{HCC}$ tissues was associated with increased age, but there were no associations with other clinicopathological features, including gender, tumor size, tumor stage, $\alpha$-fetoprotein, cirrhosis, hepatitis B surface antigen or tumor differentiation (Table II).

GATA4 and GATA5 expression reduces cell viability, inhibits colony formation and induces apoptosis of HCC cells. Since
GATA4 and GATA5 expression was reduced in HCC tissues and cell lines, the present study hypothesized that restoration of GATA4 and GATA5 expression may suppress HCC. Therefore expression vectors were constructed for GATA4 and GATA5, which were then transfected into HepG2 cells. The results of the present study revealed that overexpression of GATA4 or GATA5 in HepG2 cells (Fig. 3A) significantly reduced cell viability compared with those transfected with the control vector, as indicated by MTS assay (Fig. 3B; 30.14 and 39.31\% inhibition following 6 days of incubation, respectively; $\mathrm{P}<0.05$ ). In addition, flow cytometric analysis indicated that the cell cycle was arrested. In particular, the fraction of HepG2 cells in the G2-M phase following GATA4 and GATA5 transfection was 8.83 and $13.31 \%$, respectively, compared with $15.14 \%$ in pc-con-transfected HepG2 cells ( $\mathrm{n}=3$; $\mathrm{P}<0.05$; Fig. 3C). Furthermore, restoration of GATA4 and GATA5 gene expression inhibited the colony formation ability of HepG2 cells (Fig. 3D; $\mathrm{P}<0.05)$ compared with that of control cells. Restoration of GATA4 and GATA5 gene expression induced HepG2 cells to undergo apoptosis (20.46 and $24.79 \%$, respectively, vs. $11.28 \%$; $\mathrm{P}<0.05$; Fig. 3E).

GATA4 and GATA5 inhibit Wnt/ $\beta$-catenin activity in HCC cells. The present study also investigated whether GATA4 or GATA5 affected Wnt/ $\beta$-catenin signaling in HepG 2 cells, by performing a luciferase reporter assay. The results revealed that the luciferase 
Table II. Association of GATA5 promoter methylation with clinicopathological factors in patients with hepatocellular carcinoma.

Clinicopathological factor $\quad$ Methylated, $n(n=13) \quad$ Unmethylated, $n(n=25) \quad P$-value

Age, years

$<50$

$\geq 50$

Gender

Male

Female

$\alpha$-fetoprotein, $\mu \mathrm{g} / 1$

$<20$

$\geq 20$

Liver cirrhosis

Absent

Present

Hepatitis B surface antigen

Negative

Positive

Tumor size, $\mathrm{cm}$

$\leq 5$

$>5$

Tumor differentiation

Well

Moderate

Poor

UICC TNM ${ }^{\text {a }}$ stage

I-II

III
Methylated, n (n=13)

Unmethylated, $\mathrm{n}(\mathrm{n}=25)$
12

12

12

21

4

\section{4}

8

5

4

9

0

8

5

$8+10$

5

${ }^{a}$ Union for International Cancer Control Tumor Node Metastasis stage.

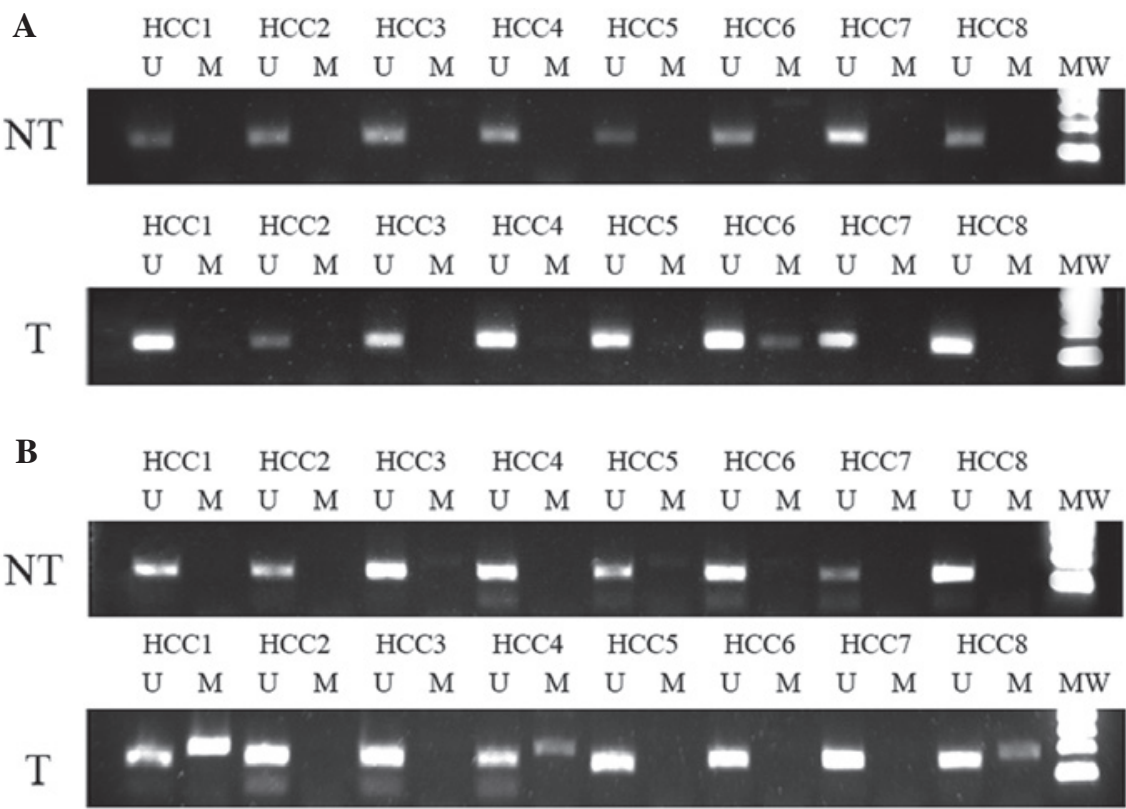

Figure 2. MSP analysis of GATA4 and GATA5 promoter methylation in HCC tumors and adjacent non-tumor tissues. (A) Representative data of MSP analysis of GATA4 in tumor tissues and paired adjacent non-tumor tissues. (B) Representative data from MSP analysis of GATA5 in tumor tissues and paired adjacent non-tumor tissues. U, unmethylated alleles; M, methylated alleles; MSP, methylation-specific polymerase chain reaction; T, tumor tissue; NT, non-tumor tissue; MW, molecular weight. 
A
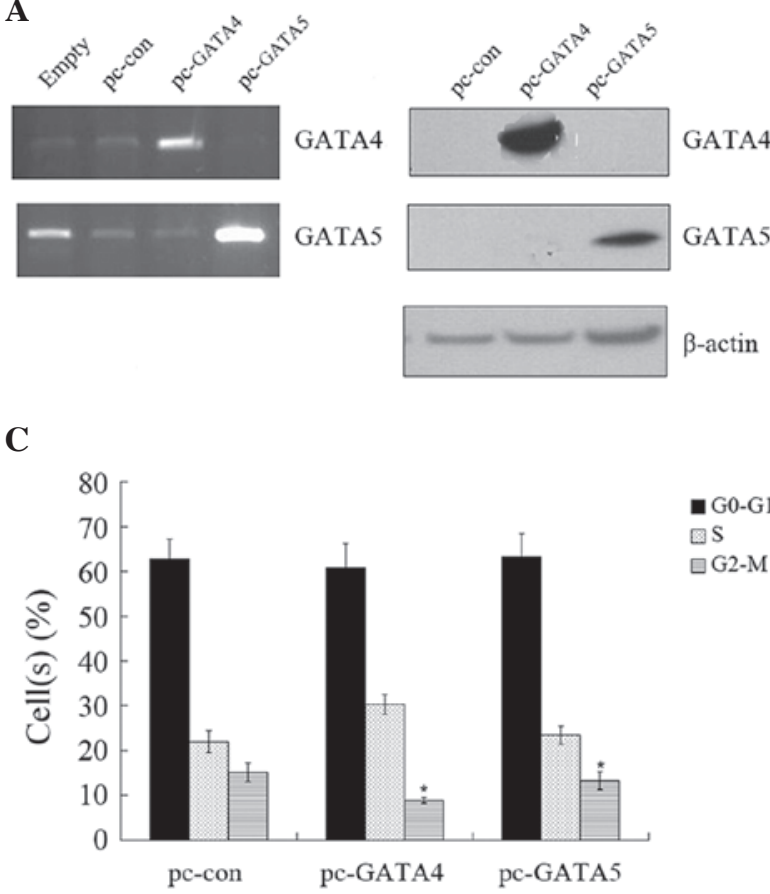

$\mathbf{E}$

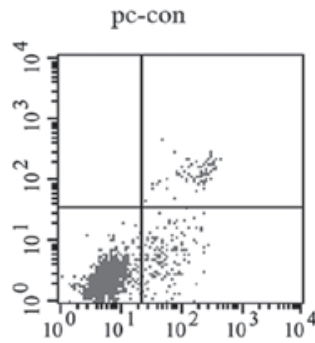

Annexin Y FITC

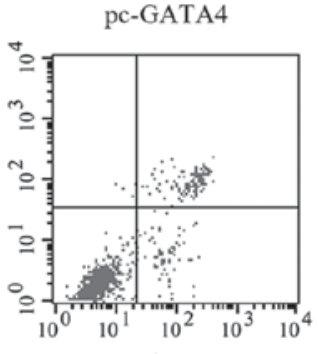

Annexin Y FITC

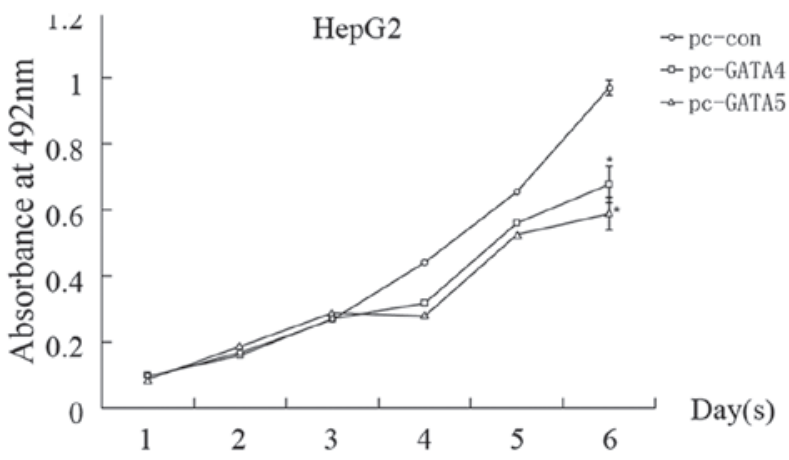

D

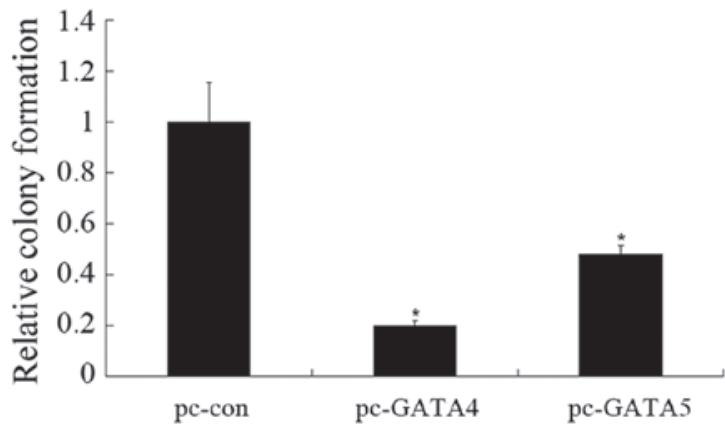

Figure 3. GATA4 and GATA5 expression reduces cell viability, inhibits colony formation and induces apoptosis in hepatocellular carcinoma cells. (A) HepG2 cells were transfected with control, pcMV-GATA4 or pcMV-GATA5, respectively for $72 \mathrm{~h}$. Total cellular RNA or protein was extracted for reverse transcription-polymerase chain reaction and western blot analyses, respectively. (B) HepG2 cells were transfected with control, pcMV-GATA4 or pcMV-GATA5 for $72 \mathrm{~h}$ and subjected to an MTS assay. (C) HepG2 cells were transfected with control, pcMV-GATA4 or pcMV-GATA5 for $72 \mathrm{~h}$ and subjected to propidium iodide staining and flow cytometry. (D) HepG2 cells were transfected with control, pcMV-GATA4 or pcMV-GATA5 for $72 \mathrm{~h}$, treated with gentamycin sulfate for $14 \mathrm{~d}$, and subsequently subjected to a colony formation assay. Quantification of colonies is expressed as the mean \pm standard error, relative to the control transfectants (pc-con) of three independent experiments ("P<0.05). (E) HepG2 cells were transfected with control, pcMV-GATA4 or pcMV-GATA5 for $72 \mathrm{~h}$ and subjected to apoptosis detection and flow cytometry. The results indicated that GATA4 and GATA5 inhibited cell proliferation, and induced cell cycle arrest and apoptosis, ${ }^{*} \mathrm{P}<0.05$. pc, pCMV vector; con, control; FITC, fluorescein isothiocyanate.

activity of TCF in pCMV-GATA4-transfected HepG2 cells was reduced compared with that of the control HepG2 cells (Fig. 4A; $\mathrm{P}<0.05)$. The luciferase activity of TCF in pCMV-GATA4-transfected HepG2 cells following co-transfection with a mutated $\beta$-catenin plasmid was significantly reduced compared with those transfected with the control vector (Fig. 4B; $\mathrm{P}<0.05$ ). GATA5-transfection luciferase reporter assays demonstrated similar results (Fig. 4C and D). However, the luciferase activity in cells co-transfected with pCMV-GATA5 and wild-type $\beta$-catenin exhibited no significant changes compared with that of the control cells (Fig. 4E), indicating that GATA4 and GATA5 inhibited the activity of the Wnt/ $\beta$-catenin pathway in HCC.

\section{Discussion}

In the current study, the expression of GATA4 and GATA5 and the methylation status of their gene promoters in HCC cells were initially determined. It was demonstrated that the expression of GATA4 and GATA5 mRNA was lost, and the GATA4 and GATA5 promoter regions were hypermethylated in certain HCC cell lines. In addition, ex vivo data revealed that the GATA5 promoter was frequently methylated in HCC tissues. Restoration of GATA4 and GATA5 expression in HCC cells significantly reduced tumor cell proliferation and colony formation, as well as inducing apoptosis. In conclusion, the results of the present study suggest that GATA4 and GATA5 may have a role in the suppression of HCC development.

A previous study revealed that loss of GATA4 and GATA5 expression was due to gene promoter methylation in gastric and ovarian cancer, which was also confirmed in lung cancer, esophageal cancer and pancreatic cancer (14-16). In the current study, it was demonstrated that the methylation of GATA4 and GATA5 promoters were in accordance with these previous studies. To the best of our knowledge, the present study is the 
A

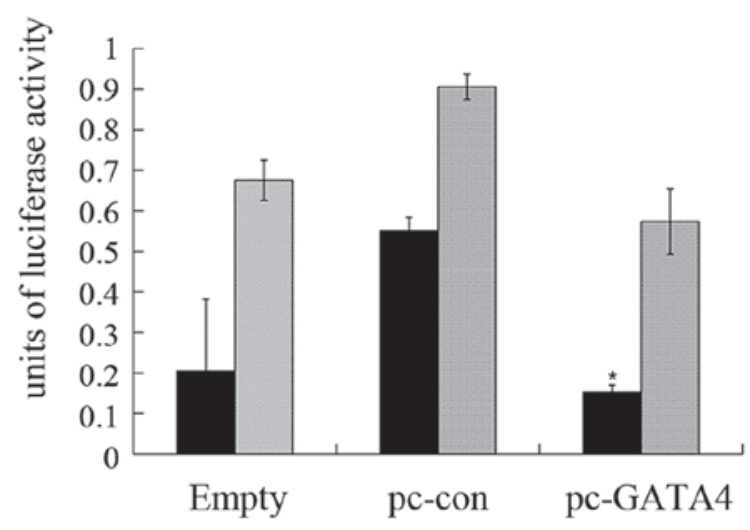

C

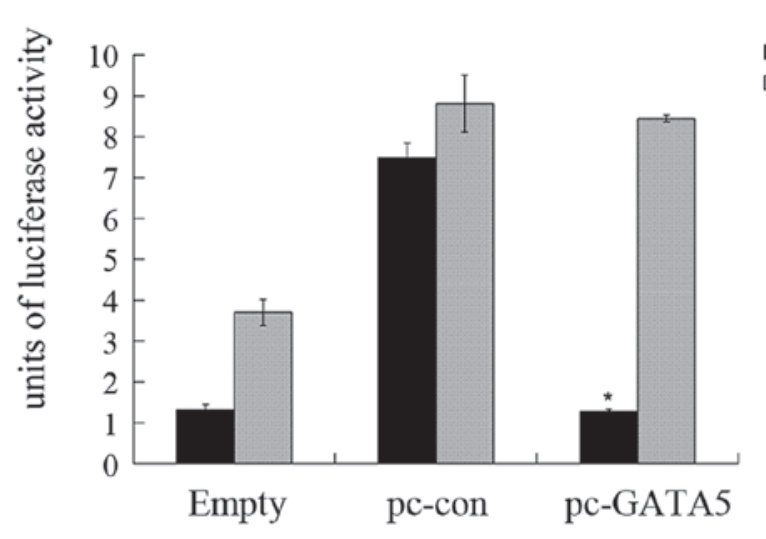

B

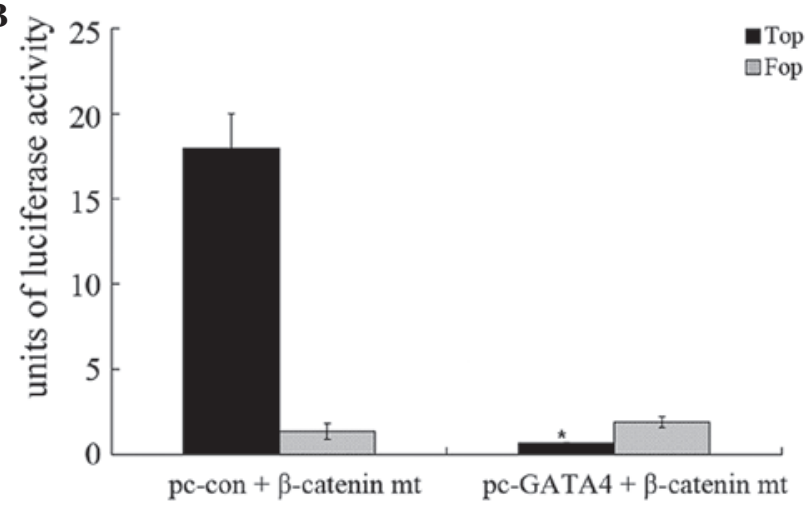

D

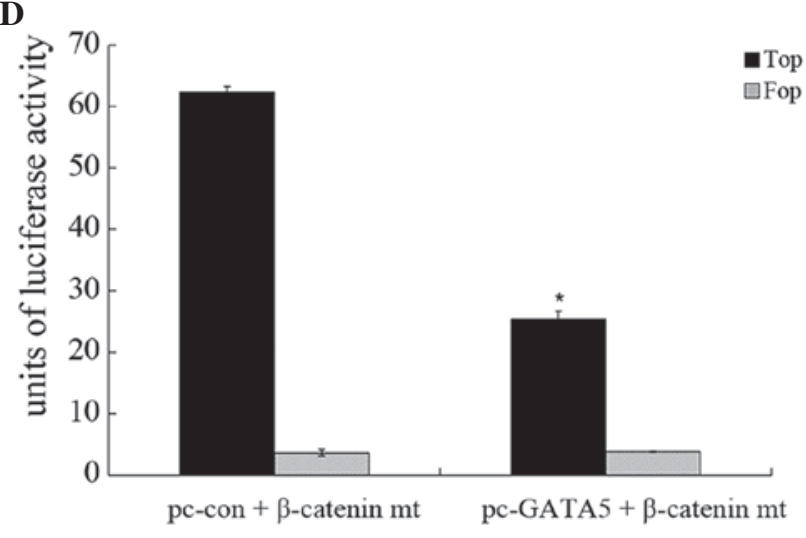

E

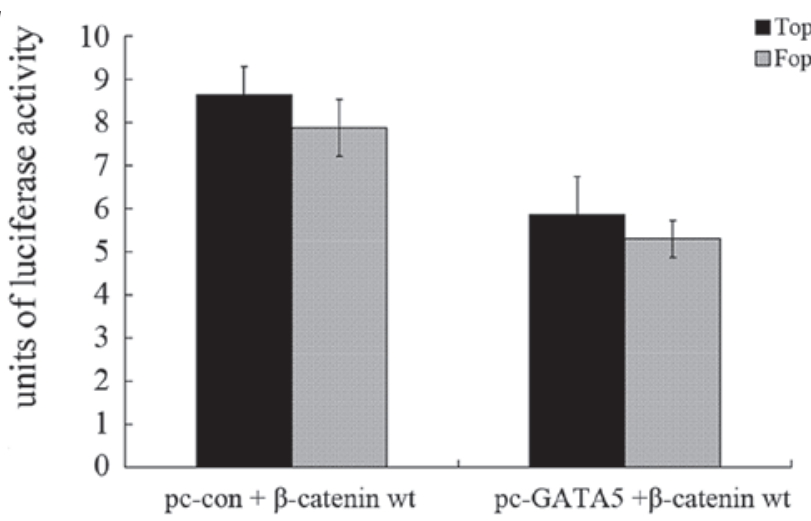

Figure 4. GATA4 and GATA5 inhibit Wnt/ $\beta$-catenin signaling in hepatocellular carcinoma cells. Luciferase reporter assay results. HepG2 cells were transfected with control, pcMV-GATA4 or pcMV-GATA5 following transfection with or without a mutated $\beta$-catenin vector. Subsequently, total cellular protein was isolated and quantified for the luciferase reporter assay. Transcriptional activity was driven by $\beta$-catenin/TCF. The basal transcriptional activity of HepG2 cells was determined by co-transfection with pGL3 and pRL-TK vectors. (A) GATA4 transfection. *P<0.05. (B) GATA4 transfection following co-transfection with or without a mutated $\beta$-catenin vector. ${ }^{*} \mathrm{P}<0.05$. (C) GATA5 transfection. ${ }^{*} \mathrm{P}<0.05$. (D) GATA5 following co-transfection with or without a mutated $\beta$-catenin vector. ${ }^{*} \mathrm{P}<0.05$ vs pCMV. (E) GATA5 transfection following co-transfection with wild type $\beta$-catenin vector. Values are expressed as the mean \pm standard error. pc-con, control vector; mt, mutant; wt, wild-type.

first investigation into GATA4 and GATA5 methylation in HCC. GATA4 was revealed to be significantly downregulated in HCC cell lines, and its promoter was hypermethylated or partially methylated in $3 / 7 \mathrm{HCC}$ cell lines. In addition, GATA5 was significantly downregulated in HCC cell lines and its promoter was hypermethylated or partially methylated in 4/7 HCC cell lines and 13/38 (34.2\%) cases of primary liver tumors. The results of the present study implied that aberrant methylation of the GATA4 and GATA5 promoter regions results in a loss of GATA4 and GATA5 expression in HCC cell lines. However, HCC cell lines that have methylated and unmethylated alleles express weak and strong levels of GATA4 and GATA5, indicating the heterogeneous population present in the cell culture. The present study also demonstrated that GATA5 promoter methylation was more frequent in older patients, indicating that GATA5 promoter methylation may be an independent risk factor for older HCC patients.

GATA4 and 5, as transcription factors, are involved in normal cell growth and also in adrenal gland, lung, ovary and colorectal cancer development (12). Functionally, they activate the promoter of tumor suppressor genes and serve as tumor 
suppressors, the lost expression of which accelerates the development of colon cancer. For example, colon cells with GATA4 and 5 expression had reduced colony formation, proliferation, migration, invasion and anchor growth (12). In a previous study, GATA4 and GATA5 expression was lost in HepG2 cells and GATA4 and GATA5 transfection experiments inhibited tumor cell growth and colony formation, but induces tumor cell apoptosis. These data indicate that GATA4 and GATA5 were able to inhibit liver cancer cell proliferation. Under the same conditions, GATA5 inhibits growth and induce apoptosis to a greater extent compared with GATA4. The present study also revealed that GATA4 and GATA5 may be involved in the regulation of the Wnt signaling pathway. Whether the expression of significant molecules in the Wnt signaling pathway, such as $\beta$-catenin, are regulated by GATA4 and GATA5 will require further investigation in future studies.

The demethylation agent 5-AZA is an effective anticancer therapy $(24,25)$. In the current study, 5-AZA treatment rescued the expression of GATA5 mRNA by reversing GATA5 hypermethylation in HCC cell lines. This finding indicated that epigenetic therapy may be useful in at least a subset of HCC patients. However, further studies are required to verify this possibility.

In conclusion, the current study demonstrated that loss of GATA4 and GATA5 expression occurred primarily due to promoter methylation in HCC tissues and cells. Restoration of GATA4 and GATA5 expression inhibited tumor cell viability and colony formation, as well as inducing apoptosis. Future studies may be required to investigate the underlying mechanisms of GATA4 and GATA5 antitumor activities in HCC.

\section{Acknowledgements}

The present study was supported in part by grants from the China National Science Foundation (grant no. 81201957) and from the National ST Major Project for Infectious Diseases of China (grant no. 2012ZX10002-017).

\section{References}

1. Parkin DM: Global cancer statistics in the year 2000. Lancet Oncol 2: 533-543, 2001.

2. El-Serag HB and Rudolph KL: Hepatocellular carcinoma: Epidemiology and molecular carcinogenesis. Gastroenterology 132: 2557-2576, 2007.

3. Thorgeirsson SS and Grisham JW: Molecular pathogenesis of human hepatocellular carcinoma. Nat Genet 31: 339-346, 2002.

4. Um TH, Kim H, Oh BK, Kim MS, Kim KS, Jung G and Park YN Aberrant $\mathrm{CpG}$ island hypermethylation in dysplastic nodules and early $\mathrm{HCC}$ of hepatitis B virus-related human multistep hepatocarcinogenesis. J Hepatol 54: 939-947, 2011.

5. Nishida N, Nagasaka T, Nishimura T, Ikai I, Boland CR and Goel A: Aberrant methylation of multiple tumor suppressor genes in aging liver, chronic hepatitis and hepatocellular carcinoma. Hepatology 47: 908-918, 2008.

6. Molkentin JD: The zinc finger-containing transcription factors GATA-4, -5 , and -6. Ubiquitously expressed regulators of tissue-specific gene expression. J Biol Chem 15: 275 , 38949-38952, 2000
7. Patient RK and McGhee JD: The GATA family (vertebrates and invertebrates). Curr Opin Genet Dev 12: 416-422, 2002

8. Laverriere AC, MacNeill C, Mueller C, Poelmann RE, Burch JB and Evans T: GATA-4/5/6, a subfamily of three transcription factors transcribed in developing heart and gut. J Biol Chem 269: 23177-23184, 1994.

9. Fujikura J, Yamato E, Yonemura S, Hosoda K, Masui S, Nakao K, Miyazaki Ji J and Niwa H: Differentiation of embryonic stem cells is induced by GATA factors. Genes Dev 16: 784-789, 2002.

10. Gao X, Sedgwick T, Shi YB and Evans T: Distinct functions are implicated for the GATA-4, -5 , and -6 transcription factors in the regulation of intestine epithelial cell differentiation. Mol Cell Biol 18: 2901-2911, 1998.

11. Akiyama Y, Watkins N, Suzuki H, Jair KW, van Engeland M, Esteller M, Sakai H, Ren CY, Yuasa Y, Herman JG and Baylin SB: GATA-4 and GATA-5 transcription factor genes and potential downstream antitumor target genes are epigenetically silenced in colorectal and gastric cancer. Mol Cell Biol 23: 8429-8439, 2003.

12. Hellebrekers DM, Lentjes MH, van den Bosch SM, Melotte V, Wouters KA, Daenen KL, Smits KM, Akiyama Y, Yuasa Y, Sanduleanu S, et al: GATA4 and GATA5 are potential tumor suppressors and biomarkers in colorectal cancer. Clin Cancer Res 15: 3990-3997, 2009.

13. Lassus H, Laitinen MP, Anttonen M, Heikinheimo M, Aaltonen LA, Ritvos O and Butzow R: Comparison of serous and mucinous ovarian carcinomas: Distinct pattern of allelic loss at distal $8 p$ and expression of transcription factor GATA-4. Lab Invest 81: 517-526. 2001.

14. Peters I, Gebauer K, Dubrowinskaja N, Atschekzei F, Kramer MW, Hennenlotter J, Tezval H, Abbas M, Scherer R, Merseburger AS, et al: GATA5 CpG island hypermethylation is an independent predictor for poor clinical outcome in renal cell carcinoma. Oncol Rep 31: 1523-1530, 2014.

15. Guo M, House MG, Akiyama Y, Qi Y, Capagna D, Harmon J, Baylin SB, Brock MV and Herman JG: Hypermethylation of the GATA gene family in esophageal cancer. Int J Cancer 119: 2078-2083, 2006.

16. Wen XZ, Akiyama Y, Pan KF, Liu ZJ, Lu ZM, Zhou J, Gu LK, Dong CX, Zhu BD, Ji JF, et al: Methylation of GATA-4 and GATA-5 and development of sporadic gastric carcinomas. World J Gastroenterol 16: 1201-1208, 2010.

17. Martin J, Afouda BA and Hoppler S: Wnt/beta-catenin signalling regulates cardiomyogenesis via GATA transcription factors. J Anat 216, 92-107, 2010

18. Afouda BA, Martin J, Liu F, Ciau-Uitz A, Patient R and Hoppler S: GATA transcription factors integrate Wnt signalling during heart development. Development 135: 3185-3190, 2008.

19. Jia Y, Yang Y, Liu S, Herman JG, Lu F and Guo M: SOX17 antagonizes WNT/ $\beta$-catenin signaling pathway in hepatocellular carcinoma. Epigenetics 5: 743-749, 2010.

20. Edge SB and Compton CC: The American Joint Committee on Cancer: The 7th edition of the AJCC cancer staging manual and the future of TNM. Ann Surg Oncol 17: 1471-1474, 2010.

21. Mayer MP: A new set of useful cloning and expression vectors derived from pBlueScript. Gene 163: 41-46, 1995.

22. Guo M, Akiyama Y, House MG, Hooker CM, Heath E, Gabrielson E, Yang SC, Han Y, Baylin SB, Herman JG and Brock MV: Hypermethylation of the GATA genes in lung cancer. Clin Cancer Res 10: 7917-7924, 2004

23. Herman JG, Graff JR, Myöhänen S, Nelkin BD and Baylin SB: Methylation-specific PCR: A novel PCR assay for methylation status of CpG islands. Proc Natl Acad Sci USA 93: 9821-9826, 1996.

24. Kristensen LS, Nielsen HM and Hansen LL: Epigenetics and cancer treatment. Eur J Pharmacol 625: 131-142, 2009.

25. Meng F, Sun G, Zhong M, Yu Y and Brewer MA: Anticancer efficacy of cisplatin and trichostatin A or 5-aza-2'-deoxycytidine on ovarian cancer. Br J Cancer 108: 579-586, 2013. 